\title{
Global Congo. Politiques et esthétiques d'une littérature (et d'une critique) mondiale
}

\author{
Silvia Riva
}

\section{OpenEdition}

\section{Journals}

Édition électronique

URL : https://journals.openedition.org/coma/6373

DOI : $10.4000 /$ coma.6373

ISSN : 2275-1742

Éditeur

Institut des textes \& manuscrits modernes (ITEM)

Référence électronique

Silvia Riva, "Global Congo. Politiques et esthétiques d'une littérature (et d'une critique) mondiale », Continents manuscrits [En ligne], 15 | 2020, mis en ligne le 15 octobre 2020, consulté le 13 janvier 2023. URL : http://journals.openedition.org/coma/6373 ; DOI : https://doi.org/10.4000/coma.6373

Ce document a été généré automatiquement le 13 janvier 2023.

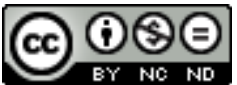

Creative Commons - Attribution - Pas d'Utilisation Commerciale - Pas de Modification 4.0 International - CC BY-NC-ND 4.0

https://creativecommons.org/licenses/by-nc-nd/4.0/ 


\title{
Global Congo. Politiques et esthétiques d'une littérature (et d'une critique) mondiale
}

\author{
Silvia Riva
}

\section{Introduction}

1 Que se passe-t-il lorsqu'on envisage la littérature congolaise non pas dans une perspective nationale, mais dans une perspective mondiale? Cette question au fondement de cette livraison de Continents manuscrits est née du constat selon lequel le Congo est souvent présenté comme l'un des épicentres de la mondialisation. Or cette dimension mondiale est habituellement envisagée uniquement du point de vue économique: on pense avant tout aux matières premières dont foisonne le pays. À l'inverse, comme un pied de nez à cette lecture monétaire du monde, nous avons décidé d'envisager la culture congolaise comme le centre d'un réseau d'échanges mondial, en considérant la littérature contemporaine du pays par le biais d'une approche littéraire récente, à savoir la world literature ou littérature mondiale. Cette approche, qui s'enracine depuis une vingtaine d'années dans les départements de Lettres aux ÉtatsUnis, peut être présentée comme une radicalisation de la littérature comparée et de ses questions : elle prend acte, d'une part, de la globalisation et se veut postnationale, et d'autre part, des critiques postcoloniales, qui reprochent aux chercheurs occidentaux, comme on le sait, leur horizon épistémologique eurocentré. Cette approche pose une série de questions qui nous semblent politiquement et esthétiquement intéressantes pour envisager une littérature comme celle du Congo.

2 Toutefois, lorsqu'on aborde les théories portant sur la littérature mondiale, on est confronté à plusieurs réflexions problématiques.

3 Tout d'abord, telle qu'elle a été envisagée pour la première fois de manière ample par Pascale Casanova notamment, cette approche se fonde sur la dichotomie «centre/ périphérie » : c'est l'idée bien connue selon laquelle les grands centres symboliques - 
Paris, Londres, New York, autrefois Bruxelles pour le Congo - attireraient tou.te.s les écrivain.e.s du reste du monde, dès lors qualifié de " périphérique ». L'unique but des écrivain.e.s serait de développer une stratégie éditoriale leur permettant de publier dans ces centres prestigieux afin d'acquérir une légitimité internationale, ou, pour le dire avec Bourdieu, une consécration. Le problème de cette grille de lecture - certes valable dans certains cas et à certaines époques - est qu'elle est, comme toute grille de lecture, réductrice. Elle a en effet tendance à empêcher les chercheur.e.s de s'intéresser à toute une production artistique qui n'entre pas dans ce schéma et qui impliquerait une recherche de terrain, extra muros académiques : il est évident que la littérature populaire, qui n'a a priori aucune vocation à s'exporter, les maisons d'éditions situées en dehors des grands circuits, les écrivain.e.s qui choisissent d'écrire dans des langues locales dites minoritaires, et les écrivain.e.s qui considèrent l'écrit comme une option d'expression artistique parmi d'autres (visuelles, musicales, plastiques, performatives) ne visent pas à intégrer un quelconque centre pour s'auréoler de son prestige. Ces acteurs et actrices ne considèrent d'ailleurs sans doute pas leur art comme "périphérique ». S'il y a une intervention culturelle périphérique, son acteur sait qu'elle fera du bien au corps (social) dans son entier: Faustin Linyekula, parmi les fondateurs des Studios Kabako disséminés sur le territoire du Congo, à Lubunga notamment, affirme qu'il fallait appliquer dans cette commune de Kisangani, centre liminaire et fragile s'il en est, un traitement sur des "points d'acuponcture qui permettent de stimuler la circulation ${ }^{1} »$.

4 Et pourtant, si leurs motivations et leurs stratégies échappent au schéma centripète, ils/ elles sont quand même connecté.e.s au monde et aux échanges internationaux qui les délocalisent et brouillent les frontières par une multitude d'entrées: les foires du livre, les réseaux sociaux, les résidences d'artistes, les festivals de théâtre et de danse, les centres culturels, les laboratoires, les studios, les ateliers créatifs, les colloques internationaux...

5 Et justement, au cours du colloque international «Global Congo, politiques et esthétiques d'une littérature mondiale » qui a eu lieu à l'Université de Milan grâce au financement d'un projet de recherche d'intérêt national (PRIN) intitulé «Mobilité stabilisation. Représentations congolaises et dynamiques sociales, au Congo et dans l'espace global ${ }^{2}$ ", nous avons tenu à renverser la question, en envisageant le Congo non pas comme une périphérie, mais comme un centre littéraire. Un centre, pour les écrivain.e.s de la diaspora d'abord, dont plusieurs contribuent à ce dossier ${ }^{3}$ : qu'ils/ elles résident en Belgique, comme Jean Bofane et Lisette Lombé, en Autriche, comme Fiston Mwanza Mujila, aux États-Unis, comme Pius Ngandu Nkashama, au Canada comme Marie Louise Bibish Mumbu et Blaise Ndala entre autres, en Allemagne comme Muepu Muamba, en Suède comme Marc-Antoine Vumilia, en Angleterre comme Norbert Mbu Mputu ou JJ Bola, ces auteur.e.s disséminé.e.s autour du globe évoquent souvent le Congo dans leurs textes et interventions, et ils/ elles y retournent de temps en temps-lorsque les conditions ne sont pas défavorables - pour développer des projets de théâtre, de danse ou d'écriture, des structures éditoriales, des ateliers, des programmes pédagogiques (comme c'est le cas pour Clémentine Faïk-Nzuji, qui prône l'enseignement des cultures fondamentales dans les écoles afin de «revoir les catégories héritées d'une époque révolue »). Tout cela nous convainc de l'existence de ce centre littéraire dont nous avons émis l'hypothèse. 
Comme l'affirme Blaise Ndala dans l'entretien «L'extension sous toutes les latitudes de "la république des lettres" » mené, comme la plupart des interviews, par Julien Jeusette qui a d'ailleurs collaboré à la conception de l'argumentaire du colloque :

Il existe bel et bien une littérature congolaise comme il existe une littérature belge réunissant sous la même bannière aussi bien une Amélie Nothomb racontant le Japon depuis les bords de Seine, que sa compatriote Geneviève Damas qui convoque la Centrafrique depuis le Plat-Pays. Que le colloque «Gobal Congo» qui nous a réunis voilà un an à Milan nous ait conviés à sa tribune, Sinzo Aanza et moi - parmi d'autres -, alors que je vis en Ontario au Canada où je suis publié, et que Aanza vit à Kinshasa où il offre au public l'essentiel de sa production littéraire, est à l'évidence le signe qu'au-delà des lieux d'où nous créons, se dessine une filiation littéraire qui conjugue des éléments aussi épars que l'origine nationale, une certaine idée d'un socle culturel commun, le théatre où nos imaginaires puisent matière à dire et à interroger le monde.

7 Un éditeur a déclaré que « se faire reconnaître par le Nord n'est pas du tout le premier enjeu ${ }^{4}$ » des écrivain.e.s africain.e.s. Ainsi, le concept de "significant geographies» développé par Francesca Orsini, professeure de Littérature Hindi et Sud-Asiatique à la SOAS de Londres ${ }^{5}$, nous semble intéressant dans la mesure où il complique la dichotomie totalisante centre/ périphérie, et tente de saisir la géographie qui compte pour les écrivains - quelles sont donc les géographies imaginaires, conceptuelles et réelles qui comptent pour les écrivains (congolais) ? Une approche de la world literature classique (à savoir, qui s'inspire de l'école états-unienne) répondrait : « les États-Unis » ou «l'Europe", mais l'étude de cette littérature nous prouve évidemment que c'est faux, et cela remet en question l'idée de « réel » par rapport à la place qu'occupe la littérature congolaise - et de beaucoup d'autres littératures non seulement africaines à l'échelon global quand on se fonde sur un mode d'approche " quantitatif », comme le fait Pierre Halen dans l'article «La littérature congolaise: une première approche quantifiée grâce à LITAF ». Il se demande : "Qu'en est-il réellement de la littérature congolaise? Non pas ce que nous voudrions qu'elle soit, mais ce qu'elle représente effectivement, en termes d'œuvres publiées et commentées? ». C'est là que réside le cœur du problème que cette livraison, au titre provocateur et totalisant, désire aborder : qu'est-ce qu'une œuvre littéraire (et pas seulement en Afrique) de nos jours ? Uniquement ce qui est publié et commenté ? Ou, plutôt, aussi ce qui est crié dans la rue, ou dans un théatre, ou dans une salle de conférence universitaire ${ }^{6}$ ? Et encore, dès lors que la dimension orale et performative, présente depuis toujours en Afrique, revient en force aujourd'hui partout, la littérature, les faits littéraires, ne risquent-ils de devenir génétiques en eux-mêmes, c'est-à-dire de devenir des littératures " d'avant-temps » et "d'avant le livre », comme semble le suggérer Nicolas Martin-Granel dans son article, riche d'enseignements, «D'une rive l'autre : questions de génétique et poétique ${ }^{7}$ » à propos des « écrivains bi-riverains » congolais?

8 Sinzo Aanza, qui nous offre ici ses « Notes d'intention pour Plaidoirie pour vendre le Congo ", son dernier spectacle théâtral, souligne la nécessité de s'exprimer dans un art total, qui prenne en compte une fois de plus le corps. Comme le rappelle Martin-Granel, Sinzo Aanza affirmait déjà dans le tract programme de l'installation Pertinences citoyennes (Galerie Imane Farès, 2018) :

Il me fallait une littérature qui donne aux mots une autre forme, un autre agencement, une littérature dont les mots sont des objets par exemple ou des sons, des surfaces et des volumes, il me fallait cette littérature-là avec des mots qu'on peut rencontrer dans la rue, des mots qui n'ont pas attendu que l'on ouvre le livre 
qui les contient. En tant qu'écrivain au Congo (je semblais être au mieux un anachronisme, au pire une farce) je n'avais pas une place au-delà de celle que quémandait mon corps dans une société où la confrontation des corps a déjà engendré des catastrophes qui attendent encore d'être nommées pour sereinement passer à la mémoire, il fallait que ma poésie soit un corps supplémentaire, un corps ou plusieurs corps en plus du mien et qui viennent, eux aussi, négocier l'espace aux côtés de mon propre corps.

Des corps parlants, des corps en mouvement sur les tréteaux, des tracts programmes, des notes d'intention, des toiles, des photos, des posts sur Facebook, des plans de villes maltraités, des textes de chanson rappés, des slams, des archives renversées, des résumés inachevés : autant de textes épars qui correspondent aux conditions mentales, mais aussi matérielles dans laquelle l'écrivain travaille au Pays. Peut-on imaginer la difficulté d'écrire quand on est interrompu continuellement par des « délestages », les coupures d'électricité ? Aucune trace, si on ne sauvegarde pas tout le temps...

Quant aux livres papier, comme l'affirme Christian Gombo qui nous conduit dans le cœur de la circulation locale dans le riche entretien mené par Claire Riffard et Sonia Le Moigne-Euzenot « Oui, le Congo est un centre littéraire », ils sont partout :

vente aux étalages de supermarchés, chez les bouquinistes où ils sont étalés par terre presque partout dans le pays, vente dans quelques restaurants bien cotés (un modèle qui a bien fait vendre mes fables), vente directe dans les écoles (Zando BD), vente ambulante organisée, vente dans les salles de fêtes (mariages, anniversaires dans les sphères riches), vente en ligne avec possibilité de livraison à domicile en exploitant les réseaux sociaux, vente de livres en format pdf via Internet avec des paiements en mobile Money, vente dans les foires agricoles, industrielles, minières... Bref, tout rassemblement peut être l'occasion de croiser des lecteurs potentiels et, une fois que ce modèle de vente sera bien organisé, ce sera une base solide sur laquelle reposera la rentabilité du livre.

Il faut donc être attentifs aux maisons d'édition qui travaillent dans les langues locales (dans le cadre de cette livraison, Nzoi et Mabiki, dont nous parlent, respectivement, Christian Gombo et Bienvenu Sene Mongaba dans l'entretien « La littérature en langues congolaises nous permet d'exister souverains»), aux auteurs qui s'expriment en langues locales (par exemple en kiswahili) ou qui ne publient pas (c'est le résultat surprenant du travail de terrain effectué par Flavia Aiello et Roberto Gaudioso sur « Les transgressions poétiques d'un auteur swahiliphone de Lubumbashi: Patrick Mudekereza »), aux stratégies éditoriales des jeunes écrivains qui se retrouvent dans de nouveaux cercles littéraires - autrefois c'était «La Pléiade du Congo » animé par Clémentine Nzuji et avec la participation de V.Y. Mudimbe, hier c'était encore le collectif Libr'écrire à Lubumbashi, né à l'initiative de l'écrivain haïtien Jean André Constant, avec Fiston Mwanza Mujila, Mudekereza, Ramcy Kabuya et Maëline Le Lay, entre autres ${ }^{8}$-, aux lecteurs idéaux (selon la célèbre formule de Gérard Genette et la magnifique image introduite par Jean Bofane dans son inédit « Le lecteur fantôme ») et aux circulations des textes (en traduction ou en langues originales) entre le Congo et le reste du monde.

De ce point de vue, on peut se référer d'abord aux relations transafricaines : la pièce de Sinzo Aanza, Plaidoirie pour vendre le Congo (2020), par exemple, a été mise en scène en septembre-octobre 2020 dans le cadre de la $11^{e}$ édition des "Récréatrales " à Ouagadougou par Aristide Tarnagda (compagnie théâtrale Acclamations), que Aanza avait rencontré dans le cadre des "Praticables " à Bamako pour la mise en scène du texte Le Jour du massacre (2018). 

d'aujourd'hui (avec le premier roman de Blaise Ndala, J'irai danser sur la tombe de Senghor, traduit en russe à partir du Québec; ou avec Marie-Louise Bibish Mumbu, basée au Canada, représentée ici par son récit inédit délibérément déstabilisant pour son cadre social et spatial de Far West et de ranch, «N'écoute pas ta mère!»), mais aussi pour les échanges entre Haïti et le Congo qui remontent bien avant la fondation de Libr'écrire en 2007: au tout début des années soixante, Elisabeth Mudimbe-Boyi, professeure émérite de Littératures françaises et comparées à l'Université de Stanford interviewée par Jean Jonassaint, fut la première chercheure congolaise à avoir introduit l'œuvre du romancier haïtien Jacques Stephen Alexis dans les universités du Congo («Des traces littéraires haïtiennes au Congo »). Grâce au récit de cette expérience pionnière, Elisabeth Mudimbe-Boyi porte un témoignage essentiel sur les échanges entre les deux rives de l'Atlantique dans le milieu académique de Kinshasa et Lubumbashi au tout début de l'Indépendance, avec la figure "anarchique » du professeur Victor Bol, et le rôle courageux et parfois futuriste joué par la maison d'éditions Mont Noir fondée par V.Y. Mudimbe dans les année soixante-dix, avec, parmi les nombreux textes parus, la traduction de l'espagnol vers le français de l'autobiographie d'un Congolais à Madrid, Francisco José Mopila, acteur de cinéma, artiste-peintre et écrivain diasporique... dans les années trente ${ }^{9}$. fouiller pour écrire une histoire différente, incroyablement plus complexe, des faits littéraires congolais et africains. Ce cheminement ne va pas nécessairement, comme c'est le plus souvent le cas, du Sud au Nord (ou du Nord au Sud), mais du Sud au Sud, voire aux Suds.

15 Il est aujourd'hui assez à la mode (académiquement parlant, parce que l'académie n'échappe pas à ce type de vents) d'abandonner le terme postcolonial en faveur du terme décolonial, souvent utilisé comme s'ils étaient synonymes. En réalité, si le terme postcolonial sanctionne un état de fait (qui, dans les années quatre-vingt, synthétisait la condition identitaire in-between de ceux qui vivaient la diaspora et ripostaient par leur plume à l'arrogance des Empires coloniaux ${ }^{10}$ ), le terme décolonial, issu du milieu épistémologique sud-américain (Walter Mignolo) et portugais (Boaventura de Sousa Santos), doit plutôt être compris comme une action, non pas identitaire mais plutôt humaine, une dynamique de décolonisation des mentalités et d'écologie de la connaissance planétaire : en déconstruisant les imaginaires, on découvre qu'il existe de nombreuses manières de vivre le monde, qui ne marient pas nécessairement les mêmes linéarités, syntaxes, temporalités, spatialités, supports pour véhiculer des messages, ou concepts, même de patrimoine ; à cet égard, la destruction rituelle des masques de la fête du Sigui dogon au Mali, qui se tient tous les soixante-dix ans ${ }^{11}$, ou, plus fréquemment, la destruction des brouillons avant-textuels, vont à contresens de l'idée patrimoniale typiquement conservatrice de l'« original », typique du Nord du monde.

C'est de cette manière que l'esthétique, et le geste critique qui l'interprète et la divulgue, devient une politique.

La présence au colloque "Global Congo. Politiques et esthétiques d'une littérature mondiale » d'un artiste reconnu tel que Sammy Baloji pour présenter son travail sur la pièce de théâtre de Joseph Kiwele, Chura Na Nyota ${ }^{12}$ (dont l'illustration que l'artiste nous a très généreusement offerte sert de présentation à ce numéro de Continents Manuscrits) montre la nécessité d'inclure dans les pages de critique littéraire les perspectives 
artistiques: dans ce cas spécifique, les documents préparatoires de l'installation qui met le doigt sur les données politiques de division ethnique communautaire imposées par la colonisation impliquent une posture critique à partir de la littérature ; Sammy Baloji est, ici, critique littéraire. De plus, les éléments urbanistiques, les références à la peinture populaire de l'école du Hangar (Elisabethville/Lubumbashi, 1946-1954) qu'il utilise, démontrent la filiation entre colonialisme et mondialisation, en outre du fait évident que l'utilisation littéraire des langues locales ne met pas nécessairement le public à l'abri des instrumentalisations politiques de la propagande.

Mais si le panorama peint jusqu'ici ne semble qu'optimiste, il ne faut jamais oublier le contexte de crise profonde d'où cette littérature prend souvent la parole, ou « ce qu'elle représente effectivement ».

Dans ce dossier on rencontre plusieurs considérations poignantes sur la situation difficile du Congo et d'une bonne partie du monde entier (mondialisé). Jean Bofane nous confie, dans "Le lecteur fantôme", que le livre a été un antidote à la guerre quand il était enfant :

Avec la guerre, papa fut mis dos au mur. Il fallait absolument transcender la morbidité ambiante et atteindre des niveaux supérieurs de pensée. Il m'emmena devant la paroi faite de livres et qui ressemblait à la montagne où vivaient Hassan ibn al-Sabbah, le vieil homme de la montagne, et ses hachischins dont m'avait parlé papa, sur la véranda, un soir, au son des grillons, des hululements de rapaces nocturnes et des sifflements de chauve-souris. Il me fit me planter devant l'autel. De lui-même, mon visage se leva vers le haut comme lors d'une assomption. Papa tendit, alors, le bras, prononça une parole pleine de sens: «Choisis!». C'était comme quand le Christ avait dit à ce type : « Lazare lève-toi ! ».

La guerre que les écrivain.e.s racontent n'est pas seulement celle de Mulele en 1964.

Muepu Muamba, poète de tous les continents parce que son regard respectueux embrasse l'Humain dans son amplitude, nous rappelle dans l'un des "Quatre poèmes " inédits présentés dans ce numéro ("Bêtise somptueuse») les faits sanglants de Sarajevo, parce que les morts des guerres sont partout les mêmes :

Je ne pleure jamais les pierres mortes

les pierres sèches ne connaissent pas

la primordiale

danse d'apprivoisement

même si l'on peut coucher une pierre

déracinée

sur une pierre

déracinée

Sarajevo

De son côté, Sinzo Aanza en arrive à affirmer que ce qui l'a amené à concevoir la pièce Plaidoirie pour vendre le Congo, c'est la saleté des morts tués. La vision de la dignité d'un cadavre l'a poussé à envisager rien de moins que la vente de son pays à Wall Street pour tenter d'en accomplir le nettoyage :

La vente du Congo vient du souvenir de la saleté de la mort, ou de l'idée que je me suis fait de la saleté de la mort jusqu'au jour où, après avoir vu, dans les conflictualités installées dans mon Kivu natal par les besoins impérieux de l'économie globalisée, uniquement des cadavres confondus à la poussière ou vautrés dans de l'herbe ou composant avec l'éparpillement d'objets quelconques, je voyais enfin un mort propre, entier, digne dans sa mort. 
Finalement, dans le résumé de son article "Les métamorphoses "congolaises" des discours littéraires" daté du 18 décembre 2018 et proposé comme sujet pour le colloque "Global Congo", Pius Ngandu Nkashama ${ }^{13}$ exprime fort bien tous les problèmes, angoisses et espoirs traduits par la création littéraire :

Les situations sociales du pays sont tellement prégnantes et prenantes qu'elles finissent par envahir totalement l'univers de la fiction. Le désespoir que l'on peut éprouver soi-même parait parfois futile, superflu, par rapport à la grande misère infligée à des millions de personnes. Il suffit seulement d'ouvrir les yeux, pour se remplir le regard des pleurs d'enfants, de la désolation des parents, de la solitude des femmes, en l'instar de celles du Docteur Mukwege.

La mort est confrontée à chaque coin de rue, et l'inquiétude s'insinue comme un enjeu de combat au-delà du sentiment de la défaite. Même à distance, des récits sont rapportés fréquemment par les voyageurs de fortune, les fables reconstruites dans des conflits actuels avec une horreur insoutenable. Et cela depuis les ruptures imposées par les masses populaires aux despotismes barbares qui avaient envahi les rêves pendant des longues années. Car à chaque fois, ils ont fait l'impasse sur les conquêtes, les nuits de splendeurs, les victoires sur les défis majeurs et sur la lâcheté, les éblouissements intérieurs.

Écrire, « engendrer des héros » au milieu du roman donne la ferme conviction que le Peuple n'avait jamais tout perdu et que ces personnages extraordinaires tapis dans le silence devaient surgir, enfin, pour magnifier le monde des lutteurs: la puissance du dire et du faire. Saisir les hommes par la ceinture et les conduire vers les chemins de la vérité.

Comment les ouvrages congolais contemporains représentent-ils la mondialisation, qui est la cause principale des guerres? Le deuxième roman de Blaise Ndala Sans capote ni kalachnikov (2017) porte justement sur ce sujet, comme d'ailleurs le roman de Bofane Congo Inc. Le testament de Bismark (2015), traduit désormais en plusieurs langues et qui montre avec sarcasme lucide la vocation de laboratoire politique mondial de l'Afrique pour le moment pour le pire, on espère bientôt pour le meilleur.

Approcher la littérature en termes de world literature suppose d'être attentif aux phénomènes de traduction et de multilinguisme. De ce point de vue, le Congo est un cas exemplaire : un grand nombre de langues s'y croisent, et sa littérature contemporaine témoigne de cette richesse. Richard Ali A Mutu, par exemple, écrit en lingala, et est traduit en anglais (voir l'entretien mené par Julien Jeusette «Écrire, une activité éminemment politique ", où le romancier rappelle, entre autres, le problème de l'octroi des visas, qui l'a empêché de rencontrer Ngugi Wa Thiong'o à l'occasion de la remise d'un prix important aux États-Unis) ; Jean Bofane et Fiston Mujila ${ }^{14}$ écrivent en français, mais leurs romans sont traduits en une dizaine de langues; JJ Bola écrit directement en anglais $^{15}$; Muepu Muamba écrit ses poèmes en français, mais ses articles en allemand ; Bienvenu Sene Mongaba défend la diffusion du lingala dans les textes scientifiques aussi ; Mudekereza s'exprime en « kiswairi » katangais. On pourrait continuer la liste.

Grâce aux interventions des multiples acteurs et actrices du fait littéraire (qui diffèrent par génération, genre, langue d'expression littéraire, lieu de prise de parole), dont ce dossier entend se mettre à l'écoute, on approchera l'espace littéraire du Congo en termes de littérature mondiale. Cela nécessite de chercher en dehors des bibliothèques nationales, de se faire aider par la fratrie et la sororité des écrivain.e.s et des artistes, abattre les cloisons étanches disciplinaires et travailler de manière collaborative, pour renoncer tant à une vision surplombante, qu'à une vision de minorisation. Autrement 
dit, il faut parfois se mettre à l'écart ${ }^{16}$, mais il faut aussi revendiquer ses connaissances pour éviter tout essentialisme.

Quelques membres de la fratrie congolaise : (de gauche à droite) Muepu Muamba, Maria Nemeth, Sinzo Aanza, Silvia Riva, Pedro Monaville, Blaise Ndala

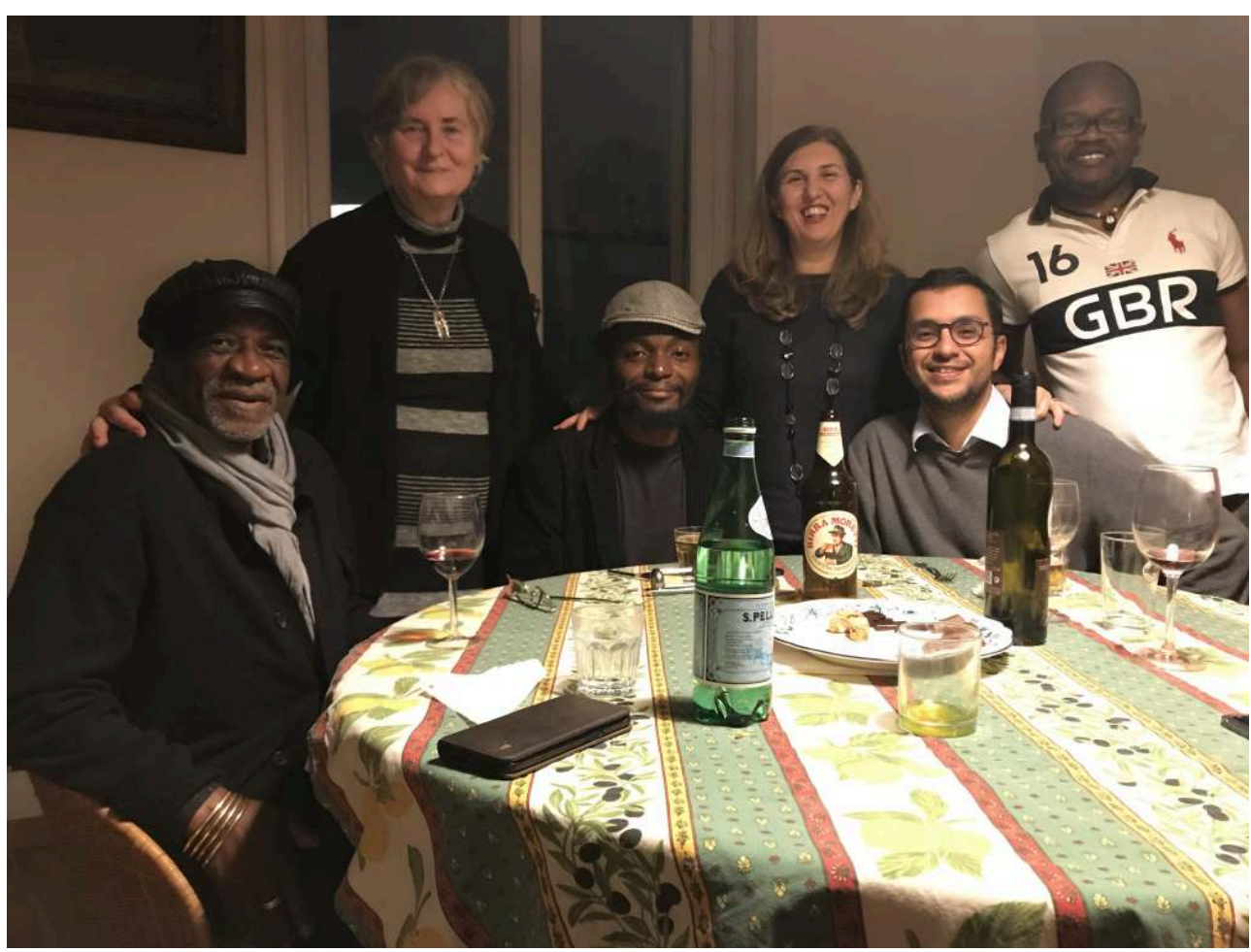

L'écoute des voix féminines dans ce dossier se fait à l'aune du lignage: «Ma mère raconte ", ainsi conclut son entretien Elisabeth Mudimbe-Boyi, tout en citant le refrain du film de Raoul Peck sur Lumumba; Clémentine Faik-Nzuji évoque les chroniques passées et récentes de son histoire familiale qu'elle nous lègue en héritage, tout comme sa vision anthropologique d'un monde où tout est relié ; Lisette Lombé, dans son slam, de manière émouvante, rappelle une femme importante de sa famille qui n'est plus, mais qui est toujours là ; elle rappelle aussi l'importance de la visibilité sur la scène de la parole « des femmes racisées, des moins valides, des personnes trans, des mamans solos, de celles qui portent le voile»; enfin Marie-Louise Bibish Mumbu crie, de manière contestataire, "N'écoute pas ta mère!" pour parler de gouvernance et de condition féminine, évidemment en pensant au Congo, mais sans se limiter géographiquement. Dans la fiction elle troque en effet "la république pour une royauté, des quartiers populaires pour un domaine, une mademoiselle-tout-le-monde pour une héritière ». Pouvoirs de la parole !

Envisager la création en acte permet ainsi d'interroger ses visées sociales et historiques : l'auteur.e entend-il/elle ainsi toucher un public plus large? Avoir un rôle plus efficace dans la société ? N'y a-t-il vraiment "Pas de poésie sans engagement ", comme l'affirme Lisette Lombé dans un entretien écrit? Y a-t-il une continuité dans l'histoire de la littérature du Congo qui dépasse la fracture coloniale et ses narratiions? Quels sont les liens qui se tissent entre générations? Quels sont les dispositifs et les outils que la littérature congolaise peut aujourd'hui mettre à la disposition de la créativité et des jeunes - public et lectorat potentiel énorme? 

la notion de reprendre comme une question de relecture (et de réécriture) de l'Afrique et de l'occident. Mudimbe utilise le terme reprendre en référence à l'art africain contemporain, pour désigner à la fois l'acte de revenir à quelque chose qui a été interrompu (littéralement re-prendre) et l'évaluation des outils à notre disposition pour le faire ${ }^{17}$. Le 30 janvier 2020 , quelques jours avant l'explosion de la pandémie en Europe, je me trouvais à Rome pour assister à une performance de Fiston Mwanza Mujila à la Villa Médicis, où Sammy Baloji était en résidence. J'ai entendu avec une stupeur et un bonheur inoubliables un kasala contemporain, accompagné de deux musiciens de jazz. Dans ce dossier, Clémentine Faïk-Nzuji évoque son intérêt pour la forme du kasala, auquel elle a consacré au début de sa carrière un «travail essentiellement sociologique, sémantique et anthroponymique [...] intitulé Analyse formelle et anthroponymique du kasala, genre poétique traditionnel luba» (1983). En 2020, à Rome, Fiston Mwanza Mujila reprenait ce chant épique traditionne ${ }^{18}$ sans interrompre le fil rouge qui était tendu depuis les temps d'avant la colonisation. L'épopée africaine est vraiment vivante ${ }^{19}$, et elle peut se rénover à chaque prise de parole. En l'espace d'une heure, le long poème de Mwanza Mujila alliait les récits cosmogoniques aux biographies réelles ou fictives, tout en évoquant d'une voix impressionnante autant par virtuosité que vigueur :

généalogies véridiques, inventées ou mises au goût du jour, fantasmes personnels $\mathrm{du}$ poète aux événements tragiques propres au Zaïre, ou encore les fosses communes de la troisième République. Encore et toujours le fleuve Congo, les creuseurs de Katekelayi, les mômes des mines du Katanga et l'Aïeul (Kaku) servant de fil rouge dans le flow du saxophone et de la basse-clarinette (Patrick Dunst), de la batterie et du gong (Christian Pollheimer) ${ }^{20}$.

Cette longue interrogation sur la nature et les implications de la relation entre langage, pensée, disciplines et dispositifs (ou outils) dans le contexte contemporain constitue la base du projet «Global Congo: politiques et esthétiques d'une littérature (et d'une critique) mondiale ».

Selon les préceptes de Citizen Science, que nous partageons, l'étude des phénomènes littéraires de nos jours ne peut que s'effectuer de manière horizontale, collaborative (c'est-à-dire en équipe, et avec la mise en commun des archives numériques des différents domaines littéraires plurilingues, ce qui pose certainement des problèmes de droits d'auteur qu'il faut envisager de manière autre). L'idée n'est pas nécessairement interculturelle : en d'autres termes, il ne s'agira en aucun cas de faire une "tournée » du Congo (ou d'ailleurs) à partir de ses littératures plurilingues, mais de retracer, par exemple, des itinéraires inattendus - comme celui des rapports entre Haïti et le Congo d'hier et d'aujourd'hui - et, par conséquent, des liens généralement négligés dans l'histoire littéraire "systémique", pour reprendre le jargon décolonial; ou bien on pourrait repérer l'absence (parlante) de relations réciproques entre certains phénomènes littéraires. 


\section{Sommaire du numéro}

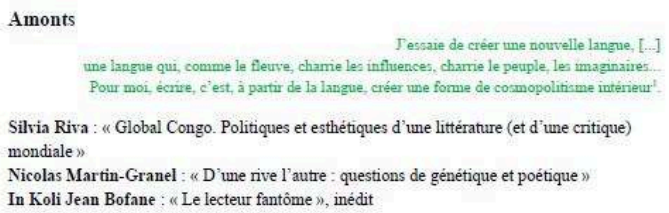

Blaise Ndala : «L'extension sous toutes les latitudes de "la république des lettres" », entretien mene par Julien Jeusette

Christian Gombo : « Oui, le Congo est un centre litteraire », entretien mené par Claire Riffard et Somia Le Moigne-Euzenot

Bienvenu Sene Mongaba : « La littérature en langues congolaises nous permet d'exister souverains ", entretien mené par Julien Jeusette

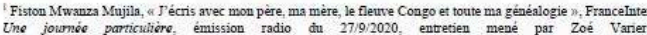

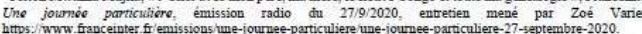
2Antoine-Roger Bolamba, « Lokolée y dans Estanzo, chans pour mon pays, Paris, présence Africaine, 1955, p. 19 3. Na Karonga ("Je rentre an pays en lingala w), Chanson de Jupiter \& Okwers, 2020. Normes et transgressions

$$
\begin{aligned}
& \text { Nen veux a personne, jet tinvite a prendre conscience, } \\
& \text { étudie beancoun, va awsil loun gue tu le pouras. } \\
& \text { remets toujiours en cause ce que tu as acquis hier. } \\
& \begin{array}{l}
\text { non pas pour te rendre insatisfait mais dans le seut souci ide faire mieus } \\
\text { non pour toi-même mais pour autriu }
\end{array}
\end{aligned}
$$

Pierre Halen : «La litterature congolaise : une premiere approche quantfièe grâce à LITAF " Flaria Aiello et Roberto Gaudioso : "Les trans gressions poétiques d' un auteur swahiliphone de Lubumbash : Patnck Mudekereza "

Marie-Louise Bibish Mumbu : «N'écoute pas ta mère ! », inédit

\section{Insoumissions}

Vois-tu, garçon, ce pays est magnifiquement lot, il y a dedans des foréts majestueuses, des nivière:

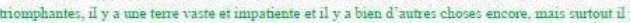
a toi et je vois dans tes yeux la même impatience cue celle de cefte tene. $j \mathrm{y}$ y vis la puissance des nivères, la mâme gràce fecconde des forêtts. Ceux qui encadrent ta vie au milieu de toute cetto purssance creatnce ont conscience de lesur fablesse et de leur indigute devant tout ça et devant tor. alors ils s' inventent ume digmite artificielle et la fécondent pour ne pass sennuver de leur propre

Muepu Muamba : "Q Quate poèmes », inédits

Lisette Lombe : « Pas de poésie sans engagement \%, entretien mené par Julien Jeuserte

activite éminemment politique $x$, entretien mené par Julien

Jro Anza : Noted' intention pour Plaidoirie pour vendre le Congo », inédit 


\section{NOTES}

1. https://currystonefoundation.org/practice/studios-kabako/; cit. dans Silvia Riva, "World Literature dans le paysage africain : le cas du Congo RD ", dans R. Giordano, E. Quaretta, D. Dibwe (dir.), Dynamiques sociales et représentations congolaises (RD Congo). «L'expérience fait la différence ", volume hommage à Bogumil Jewsiewicki, avec une postface de N.R. Hunt, Paris, L'Harmattan, 2019, p. 143.

2. Financé pour la période 2017-2020 par le ministère de l'Éducation, de l'Université et de la Recherche italien, le projet 2015 JSSXC4 a été coordonné par l'équipe de l'Université de Calabre (dirigée par R. Giordano), avec la participation de trois unités, deux italiennes et une congolaise : l'unité de l'Université de Lubumbashi (dirigée par D. Dibwe Dia Mwembu), l'Université de Naples «L'Orientale » (unité dirigée par F. Aiello), l'Université de Milan (unité dirigée par S. Riva). Voici le lien au site du colloque : https://www.globalcongo2019.it/.

3. Un autre volet des interventions sera publié prochainement sur la revue Journal of World Literature (Brill), vol. 6 (2021) : https://brill.com/view/journals/jwl/jwl-overview.xml.

4. Sulaiman Adebowale, fondateur en 2008 des éditions Amalion au Sénégal. Source de la citation: https://www.lemonde.fr/afrique/article/2018/02/09/oui-la-litterature-africaine-sedite-aussi-en-afrique_5254472_3212.html.

5. Karima Laachir, Sara Marzagora, Francesca Orsini, «Multilingual Locals and Significant Geographies: For a Ground-up and Located Approach to World Literature », 2018, Modern Languages Open, $\mathrm{n}^{\circ} 1, \quad$ p. 19: https://www.modernlanguagesopen.org/articles/10.3828/ mlo.v0i0.190/.

6. Voir le slam dans la vidéo « Slameuse, poétesse, écrivaine, féministe : Lisette Lombé ».

7. Formule qu'il emprunte à son tour à Lye M. Yoka. Voir « D'une rive l'autre: questions de génétique et poétique ».

8. Voir https://www.centredartwaza.org/?portfolio=librecrire.

9. Francisco José Mopila, L’Enfance [1 $1^{\text {re }}$ éd.: Memorias de un Congolés, ensayo de autobiographia, Madrid, Instituto des Estudio Africanos, Consejo Superior de Investigaciones Cientificas, 1949], traduction française par Jaime Castro-Segovia et Jacques Lanotte, Mont Noir, 1972.

10. Bill Ashcroft, Gareth Griffiths, Helen Tiffin, The Empire Writes Back: Theory and Practice in PostColonial Literatures, Londres et New York, Routledge, 1989; traduit en français en 2012 par les Presses Universitaires de Bordeaux sous le titre problématique L'Empire vous répond, où l'on perd totalement le jeu de mot belligérant (fight/write back).

11. Jean Rouch et Germaine Dieterlin, Sigui synthèse (1967-1973). L'invention de la parole et de la mort, Éditions Montparnasse, https://www.youtube.com/watch?v=Dy88FMbaIGo.

12. Chura na Nyoka (La Grenouille et le Serpent, 1952), publié à Elisabethville (nom de l'actuelle Lubumbashi), est une comédie musicale inspirée d'une histoire écrite par Joseph Kiwele, organiste, compositeur, professeur de musique au séminaire et engagé dans la politique. Élu député provincial, il sera nommé ministre de l'Éducation dans le gouvernement provincial du Katanga. Il a composé entre autres l'hymne national du Katanga en 1960. https:// www.zammagazine.com/chronicle/chronicle-36/666-sammy-baloji-it-s-not-yet-history

13. Pius Ngandu Nkashama (Mbujimayi 1946-) : professeur de Langues et Littératures françaises et francophones à l'université d'État de Louisiane, il est un des romanciers les plus importants et les plus politiques - à ses dépens - de la deuxième génération d'écrivains (la première étant celle de Lomami Tchibamba et Antoine-Roger Bolamba), chercheur infatigable qui, déjà dans les années quatre-vingt, défendait par son travail de romancier et de critique les droits de cité de l'écriture en langues locales (pour lui le ciluba), et sans lequel la littérature congolaise ne pourrait pas être si mondiale. 
14. Fiston Mwanza Mujila est né à Lubumbashi en 1981. Il écrit de la poésie, de la prose et du théâtre. Il vit à Graz (Autriche). Son premier roman, Tram 83, a été sélectionné pour le prix international Man Booker. Il a également reçu le prix Etisalat de littérature, la médaille d'or de littérature aux $\mathrm{VI}^{\mathrm{es}}$ Jeux de la Francophonie à Beyrouth, et le Internationaler Literaturpreis from Der Haus der Kulturen der Welt. Son deuxième roman, La Danse du Vilain (2020) vient de sortir toujours chez Métaillié (Paris), une maison d'éditions qui compte « 1000 titres qui peuvent nous ouvrir le monde à travers la diversité de la littérature et des idées, au-delà des cultures dominantes » : https://editions-metailie.com/la-maison/.

15. JJ Bola est un écrivain et poète britannique né à Kinshasa, mais basé à Londres depuis l'âge de six ans. Il a écrit quatre recueils de poésie (Elevate, Daughter of the Sun, Word, Refuge) ainsi qu'un roman, No Place to Call Home: Love, Loss, Belonging (2017), et un livre de non-fiction pour les jeunes sur la masculinité et le patriarcat, Mask Off: Masculinity Redefined (2019). Pour une lecture du poème No Place to Call Home, voir https://www.youtube.com/watch?v=QK-6EnYCwgg.

16. De ce point de vue, il est important de citer la fin de l'article de l'écrivain, critique et éditeur de plusieurs ouvrages congolais (notamment de Lomami Tchibamba) Jean-Pierre Orban, paru dans le $\mathrm{n}^{\circ} 2$, hiver 2020, de la revue Ulenspiegel (dossier « D'un Congo l'autre »), où il affirme en conclusion: «Oui, fermons, fermez, trop braves gens, musées, délégations, instituts de coopération, arrière-postes d'une histoire qui s'efface peu à peu comme un vieux tissu élimé. Et remisons nos tourments et nos amendes, honorables ou pas, dans nos greniers. L'Afrique n'en a ou ne devrait en avoir que faire. Et puisqu'il est ici surtout question du Congo, décongolisonsnous ! ». Voir http://www.cep-editions.com/livre.php?id_livre=58.

17. V.Y. Mudimbe, " "Reprendre". Énonciations et stratégies dans les arts africains contemporains ", dans Marc Quaghebeur et Émile Van Balberghe (dir.), Papier blanc, encre noire. Cent ans de culture francophone en Afrique centrale (Zaïre, Rwanda et Burundi), Bruxelles, Labor, coll. « Archives du Futur », 1992, 2 vol., XCIV-690 p., ill. ; t. II, p. 491-517.

18. Sur la feuille de salle de la performance Abattoir des rêves ou la faute à Bende, l'Aîné des Hommes, d'avoir boycotté la création? (Texte : Fiston Mwanza Mujila ; création artistique : Sammy Baloji), s.d., s.l., bilingue (fr./it.), on peut lire dans le Glossaire: «Kasala: poème de louange luba à destination de soi-même, d'un proche, ou d'un dignitaire ou même d'un personnage fictif ».

19. Robert Cornevin, "Les poèmes épiques africains et la notion d'épopée vivante ", Présence Africaine, $\mathrm{n}^{\circ}$ 60, 1966/4, p. 140-145.

20. https://www.villamedici.it/fr/les-jeudis-de-la-villa/abattoir-des-re\%CC\%82ves-ou-la-faute-abende-lai\%CC\%82ne-des-hommes-davoir-boycotte-la-creation/

\section{RÉSUMÉS}

Dans cet article introductif à la lecture du dossier «Global Congo » et aux nombreuses interventions qui le composent, Silvia Riva explore la valeur multi-sémantique du mot " global », tout en renversant les termes de la dialectique centre/périphérie et afin de mettre en valeur l'utilité critique et gnoséologique d'une approche collaborative (écrivain.e.s, critiques, artistes) et du décloisonnement des barrières disciplinaires qui s'ensuit.

In this introductory article to the reading of the "Global Congo" issue and the numerous interventions that make it up, Silvia Riva explores the multi-semantic value of the word " global », while reversing the terms of the centre/periphery dialectic and in order to highlight 
the critical and gnoseological usefulness of a collaborative approach (writers, critics, artists) and the ensuing decompartmentalisation of disciplinary barriers.

\section{INDEX}

Mots-clés : littérature mondiale, Citizen Science, Open Science, approche globale, littérature congolaise, géographies significatives

Keywords : World literature, Citizen Science, global approach, Open Science, Congolese literature, significant geographies

\section{AUTEUR}

\section{SILVIA RIVA}

Silvia Riva est professeure à l'Université de Milan. Ses domaines de recherche comprennent les littératures françaises et francophones dans leurs rapports avec les arts visuels, la philosophie, l'histoire, l'anthopologie et l'histoire des théories littéraires. Elle est l'auteure de nombreuses publications dont, récemment, « Pour une ethnologie du présent en littérature. Diffraction et recomposition du patrimoine africain francophone " (Journal des Anthropologues, $\mathrm{n}^{\circ}$ 148-149, «LittéRATURES \& Sciences sociales en quête du réel », 2017, p. 203-224 : https:// journals.openedition.org/jda/6665) et du volume Nouvelle Histoire de la littérature du CongoKinshasa (Paris, L'Harmattan, 2006, coll. « L'Afrique au cœur des Lettres »). Elle défend l'utilité des approches de Citizen Science et de la mise en commun des résultats de la recherche selon une pratique collaborative, anti-élitaire et humaniste. 